\title{
Detection of Attomole Amounts of Analyte by Desorption Electrospray Ionization Mass Spectrometry (DESI-MS) Determined Using Fluorescence Spectroscopy
}

\author{
Michael S. Bereman and David C. Muddiman \\ W.M. Keck FT-ICR Mass Spectrometry Laboratory, Department of Chemistry, North Carolina State \\ University, Raleigh, North Carolina 27695, USA
}

We report the use of fluorescence spectroscopy to investigate the amount of material removed from a PTFE surface and detected during desorption electrospray ionization (DESI) mass spectrometry measurements. The fluorescence intensity before and after DESI analysis of rhodamine 6G is used to determine the amount of material removed from the surface per mass spectrum. Calculations indicate low attomole amounts are removed per linear ion trap mass spectrum. (J Am Soc Mass Spectrom 2007, 18, 1093-1096) (c) 2007 American Society for Mass Spectrometry

$\mathrm{M}$ ass spectrometry has progressed significantly in the last few decades and this growth is primarily the result of advances in mass analyzer technology, introduction of novel ionization methods, and dissociation techniques. The development of electrospray ionization $[1,2]$ by Fenn and coworkers and matrix-assisted laser desorption ionization (MALDI) [3, 4] in the mid-1980s allowed for the first time the interrogation of biological macromolecules. However, as science advances and new problems emerge, the inherent limitations of these techniques have warranted the continued development of alternative ionization methods.

Recently several new ionization techniques-referred to in the literature as "direct analysis methods" - have been introduced. These techniques offer unique advantages because they allow for ambient analysis and require minimal sample preparation leading to higher throughput. Atmospheric-pressure solids analysis probe (ASAP) developed by Larsen and coworkers [5] and direct analysis in real time (DART) introduced by Cody et al. [6] are both techniques with a potential for a range of applications for small molecule analysis; however, to date, there are few reports describing applications of these techniques. Introduced most recently are electrospray-assisted laser desorption ionization (ELDI) $[7,8]$ and matrix-assisted laser desorption electrospray ionization (MALDESI) [9, 10]. Both techniques have exhibited potential for proteomic analyses.

Address reprint requests to Dr. David Muddiman, W.M Keck FT-ICR Mass Spectrometry Laboratory, Department of Chemistry, North Carolina State University, Raleigh, NC 27695. E-mail: david-muddiman@ncsu.edu
Developed by Cooks and coworkers in 2004, desorption electrospray ionization (DESI) [11] was one of the first techniques introduced in this "family" of direct analysis methods. Currently the majority of the direct analysis literature focuses on this technique and its applications ranging from small molecule to proteomic analyses [12]. This ionization method has been coupled to several different mass analyzer technologies, including linear ion trap [11], orbitrap [13], Fourier transform ion cyclotron resonance [14], and other hybrid MS platforms [15-17].

DESI has proven its versatility in the ability to analyze a range of analytes; however, certain fundamentals of the process remain unknown. In future biological applications of this ionization method, it is essential to explore and understand the detection limits of this technique, including the amount of material initially required and the amount subsequently removed from the surface during analysis. Cooks and coworkers have used a "down spotting technique" in which decreasing amounts of material are spotted onto a surface until no signal is obtained to report limits of detection for a range of molecules [18]. Although this technique is quick and gives a rough estimate of the detection limits of the method, it is not quantitative because the majority of sample is often left unperturbed on the surface after DESI analysis.

We report the development of a method using the fluorescence of rhodamine $6 \mathrm{G}$ to quantify the amount of material removed from the surface after $2 \mathrm{~min}$ of DESI-MS analysis. Derived from this information, calculations indicate detection of attomole amounts of the dye was achieved. It should be noted that fluorescence 
spectroscopy was previously used in conjunction with DESI to illustrate the direct analysis of species separated by TLC [15] along with the imaging capabilities of DESI-MS combined with an automated surface sampling system [19].

\section{Experimental}

\section{Materials}

Rhodamine 6G and formic acid were obtained from Sigma-Aldrich (St. Louis, MO, USA) and used without further purification. Methanol, acetonitrile, and water were purchased from Burdick \& Jackson (Muskegon, MI, USA). Polytetrafluoroethylene (PTFE) surfaces, used in these experiments, were acquired from McMaster Carr (Atlanta, GA, USA).

\section{Methods}

The DESI spray solution consisted of acetonitrile and water $(1: 1 \mathrm{vol} / \mathrm{vol})$ with $0.1 \%$ formic acid. Rhodamine 6G stock standard solutions were prepared in the same solvent composition and $10 \mu \mathrm{L}$ of a $0.02 \mu \mathrm{M}$ solution (200 fmol) was placed onto a PTFE surface and dried overnight in the ambient environment before fluorescent measurements and DESI-MS analysis. Identical amounts of rhodamine 6G (200 fmol) were deposited on all substrates. The fluorescent intensities of four samples were examined before and after DESI-MS analysis. Each sample was analyzed by DESI-MS for $2 \mathrm{~min}$.

\section{Control Experiments}

In addition, three controls were used that underwent all processes of the samples (such as physical handling, length of light exposure) except DESI analysis. The controls were prepared by the same method described for the samples. Identical amounts of rhodamine 6G were deposited on both the control and sample substrates $(200 \mathrm{fmol})$. The controls allowed for a correction in the final fluorescent intensities of the samples.

\section{DESI-MS}

Modeled after the Prosolia DESI protype [18], ${ }^{\circ}$ the desorption electrospray ionization source consisted of a $360^{\circ}$ rotational stage mounted onto an XYZ stage affording $1 \mathrm{in}$. of travel in all directions. The DESI source itself was a 1/16-in. Swagelok $\mathrm{T}$ and was affixed to the rotational stage by a plastic holder and secured by a set screw where high voltage was applied. This allowed precise adjustments of the $d_{1}$ distance $(2 \mathrm{~mm})$ and $\alpha$ angle $\left(54^{\circ}\right)$, critical for optimal signal abundance. The $d_{2}$ distance and $\beta$ angle were $<1 \mathrm{~mm}$ and $\sim 5^{\circ}$, respectively. The DESI emitter tip to MS capillary distance was $\sim 4 \mathrm{~mm}$. The DESI spray solution was infused at 2 $\mu \mathrm{L} / \mathrm{min}$ through a fused silica capillary (50 $\mu \mathrm{m}$ i.d., 150 $\mu \mathrm{m}$ o.d.). Measured at the regulator, the nitrogen pres- sure was 110 psi and flowed through an outer fused silica capillary (250 i.d., 350 o.d.) about $15 \mathrm{~mm}$ long. The inner liquid capillary extended $0.5 \mathrm{~mm}$ from the end of the gas capillary.

All mass spectra were acquired on an LTQ linear ion trap mass spectrometer (Thermo Finnigan, San Jose, CA, USA) in the positive-ion mode. The maximum trapping time was set to $200 \mathrm{~ms}$ and three microscans were obtained per mass spectrum. Automatic gain control (AGC) was set to $1 \times 10^{6}$ and never reached this target value, effectively allowing the ion trap to collect for $200 \mathrm{~ms}$ per mass spectrum. The source voltage and capillary voltage were kept constant through all experiments at $4 \mathrm{kV}$ and $37 \mathrm{~V}$, respectively. The capillary temperature was set at $200{ }^{\circ} \mathrm{C}$. Before interpretation, all selected ion chromatograms (SICs) were boxcar smoothed $(n=7)$.

\section{Fluorescence Measurements}

A BioRad Pharos FX ${ }^{\mathrm{TM}}$ Plus Molecular Imager (Hercules, CA, USA) was used to obtain the total fluorescent intensity of rhodamine 6G before and after DESI-MS analysis. The decrease in total fluorescence was then used to quantify the amount of material removed from the surface. Excitation of the dye was achieved through an internal laser (532 nm). Data were acquired and analyzed by Quantity One software provided by BioRad.

\section{Results and Discussion}

Displayed $^{\circ}$ in $^{\circ}$ Figure $^{\circ} 1^{\circ}$ are $^{\circ}$ representative $^{\circ} \operatorname{SICs}^{\circ}(\mathrm{m} / \mathrm{z}=$ $443 \pm 1$ and $415 \pm 1$ ) from the analysis of rhodamine $6 \mathrm{G}$ by DESI-MS. Confirmed by MS/MS experiments (data not shown) and possibly resulting from thermal degradation inside the mass spectrometer inlet capillary, the ion at $m / z 415$ was determined to be a fragment of rhodamine ${ }^{\circ} 6 \mathrm{G}^{\circ}{ }^{\circ} \mathrm{As}^{\circ}$ shown $^{\circ}$ in $^{\circ}$ Figure $^{\circ} 1$, the ${ }^{\circ}$ abundance ${ }^{\circ}$ of both ions track each other throughout the 2-min analysis. The inset shows three representative mass spectra obtained at $0.1,0.4$, and $1.5 \mathrm{~min}$, each collected with a trapping time of $200 \mathrm{~ms}$.

In all samples, the most abundant signal occurred between 0.1 and $0.2 \mathrm{~min}$. This delay is attributed to the initial "solvation" of the sample on the surface. As expected, the signal-to-noise $(\mathrm{S} / \mathrm{N})$ ratio decreased with increasing analysis time; however, the molecular ion at $m / z=443$ and the fragment ion at $m / z=415$ were always present throughout the 2-min analysis.

Figure $^{\circ} 2^{\circ}$ illustrates $^{\circ}$ the $^{\circ}$ fluorescent $^{\circ}$ data $^{\circ}$ obtained from the samples before and after DESI analysis. Observed was a decrease in intensity not only in the samples (S1, S2, S3, S4), but also in the three controls. The controls underwent the same physical processes as the samples (such as handling, length of light exposure) except analysis by DESI-MS. The decrease in fluorescent intensity of the controls was not as significant as the decrease as a result of DESI-MS analysis. Thus, the 


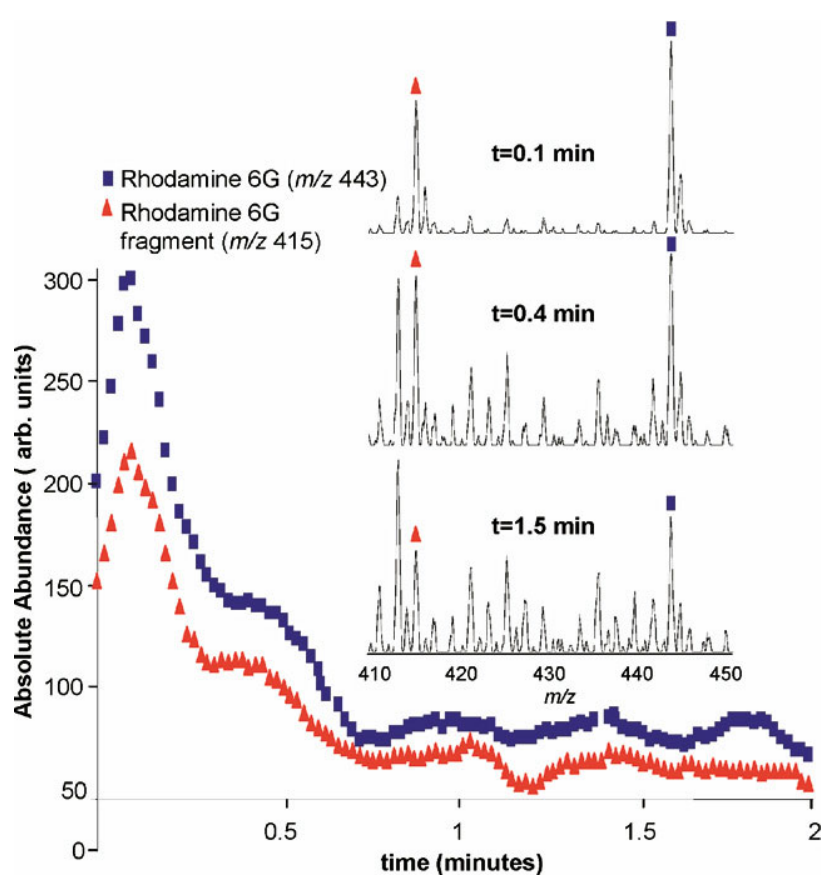

Figure 1. Selected ion chromatograms corresponding to rhodamine 6G $(m / z=443 \pm 1)$ and fragment $(m / z=415 \pm 1)$ analyzed by DESI-MS for a 2-min period (sample 4). Both ions tracked each other throughout the analysis and the $\mathrm{S} / \mathrm{N}$ decreases as a function of increasing analysis time. Three representative mass spectra are also shown at $0.1,0.4$, and $1.5 \mathrm{~min}$.

fluorescence of each of the four samples after DESI-MS analysis was increased by $9.3 \%$ (average percentage decrease of the three controls) of the initial fluorescent intensity ${ }^{\circ}$ to $^{\circ}$ account $^{\circ}$ for $^{\circ}$ this ${ }^{\circ}$ observation $^{\circ}$ (Figure ${ }^{\circ}$ ).

The total amount of material placed on the surface (200 fmol) along with the adjusted percentage decrease in fluorescence intensity allowed for a calculation of the total amount of material removed from the surface after 2 min of analysis by DESI-MS. Furthermore, using the amount of material removed in conjunction with the length of the analysis (120 s) an average amount of material removed per second can be calculated. Finally, combined with the collection time in the ion trap (200 $\mathrm{ms}$ ) and the previous calculation an average amount of material per mass spectrum can be determined. The average of all four samples indicates approximately 70 attomoles of rhodamine 6G were detected per mass spectrum. ${ }^{\circ}$ The ${ }^{\circ}$ table ${ }^{\circ}$ in ${ }^{\circ}$ Figure ${ }^{\circ}{ }^{\circ}$ summarizes ${ }^{\circ}$ these ${ }^{\circ}$ data. The material per mass spectrum is a conservative calculation and in reality this number is much lower, simply because of the sampling efficiency of DESI-MS,

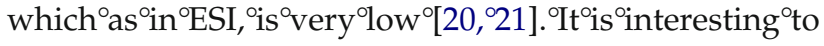
note that the amount of material removed from the surface varies by twofold. This phenomenon is attributed $^{\circ}$ to $^{\circ}$ the ${ }^{\circ}$ nonuniform ${ }^{\circ}$ distribution ${ }^{\circ}$ of $^{\circ}$ the ${ }^{\circ}$ dye $^{\circ}$ on ${ }^{\circ}$ the surface, ${ }^{\circ}$ referred ${ }^{\circ}$ to $^{\circ}$ as $^{\circ}$ the ${ }^{\circ}$ sweet-spot ${ }^{\circ}$ effect $^{\circ}[18]$.

A strong correlation $\left(R^{2}=0.988\right)$ exists between the integrated areas of the selected ion chromatograms $(\mathrm{m} / \mathrm{z}$ $443+415)$ and the amount of material removed from the ${ }^{\circ}$ surface ${ }^{\circ} s^{\circ}$ shown $^{\circ}{ }^{\circ}{ }^{\circ}$ Figure $^{\circ} 3 .^{\circ}$ This $^{\circ}$ plot $^{\circ}$ emphasizes the ability of using fluorescence to monitor the amount of material removed because a larger decrease in fluorescence (increase in material removed) directly corresponds to an increase in signal abundance.

Fluorescent pictures of rhodamine $6 \mathrm{G}$ before and after $^{\circ} \mathrm{DESI}^{\circ}$ analysis ${ }^{\circ}$ are $^{\circ}$ also ${ }^{\circ}$ displayed $^{\circ}$ in $^{\circ}{ }^{\circ}$ igure ${ }^{\circ} .^{\circ} \mathrm{As}$ previously mentioned, the rhodamine $6 \mathrm{G}$ spot varied significantly in concentration across the PTFE surface and the amount of material removed during MS analysis depended entirely on where the DESI spray impinged on the sample spot. Moreover, the areas of the rhodamine $6 \mathrm{G}$ spot on the surface ranged from 2 to 8 $\mathrm{mm}^{2}$ and greater MS signal abundance was observed from smaller spot sizes, attributed to the greater amount of material per unit area present on the surface. By use of the BioRad software and the fluorescent pictures of the samples before and after DESI-MS analysis, the diameter of the DESI spot was determined to be about $800 \mu \mathrm{m}$ for these experiments.

\section{Conclusions}

Through the coupling of two orthogonal techniquesfluorescence spectroscopy and DESI-MS - an effective method has been developed to directly determine the amount of material removed from a surface. This method allows for a calculation of the amount of analyte represented per mass spectrum. Rhodamine 6G

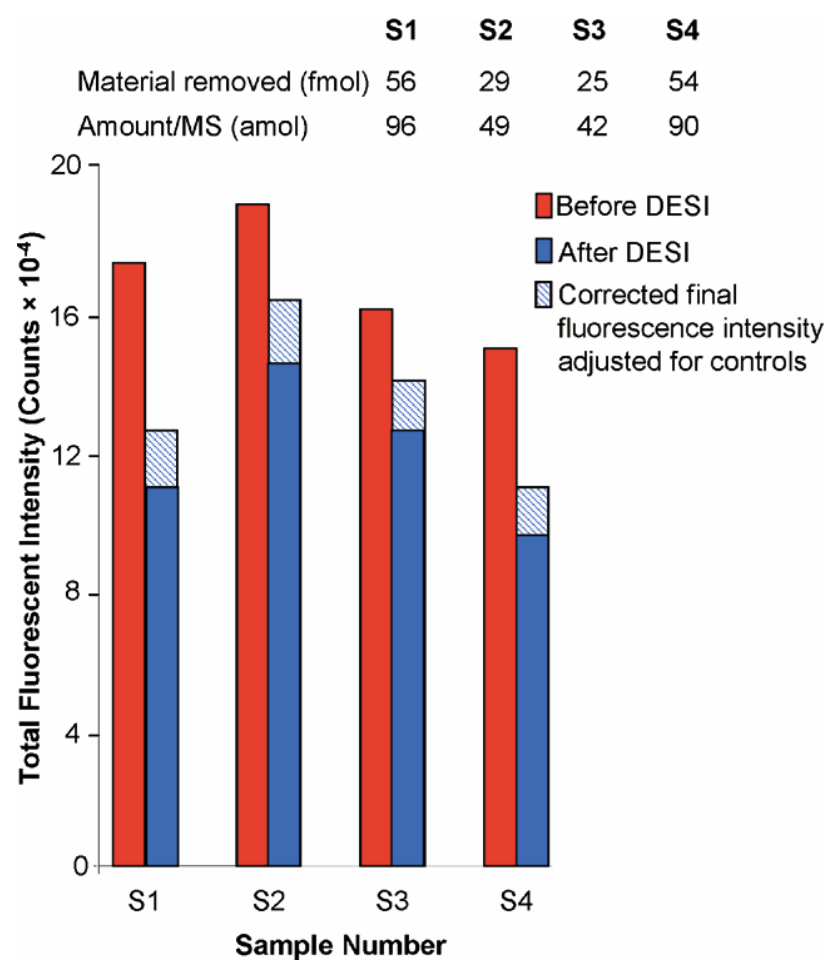

Figure 2. Comparisons in fluorescence intensities of samples before and after analysis by DESI-MS. After DESI-MS analysis, sample intensities were corrected for the average percentage decrease in the controls $(9.3 \%)$. The table inset summarizes the amount of material removed and material per mass spectrum for each sample. 


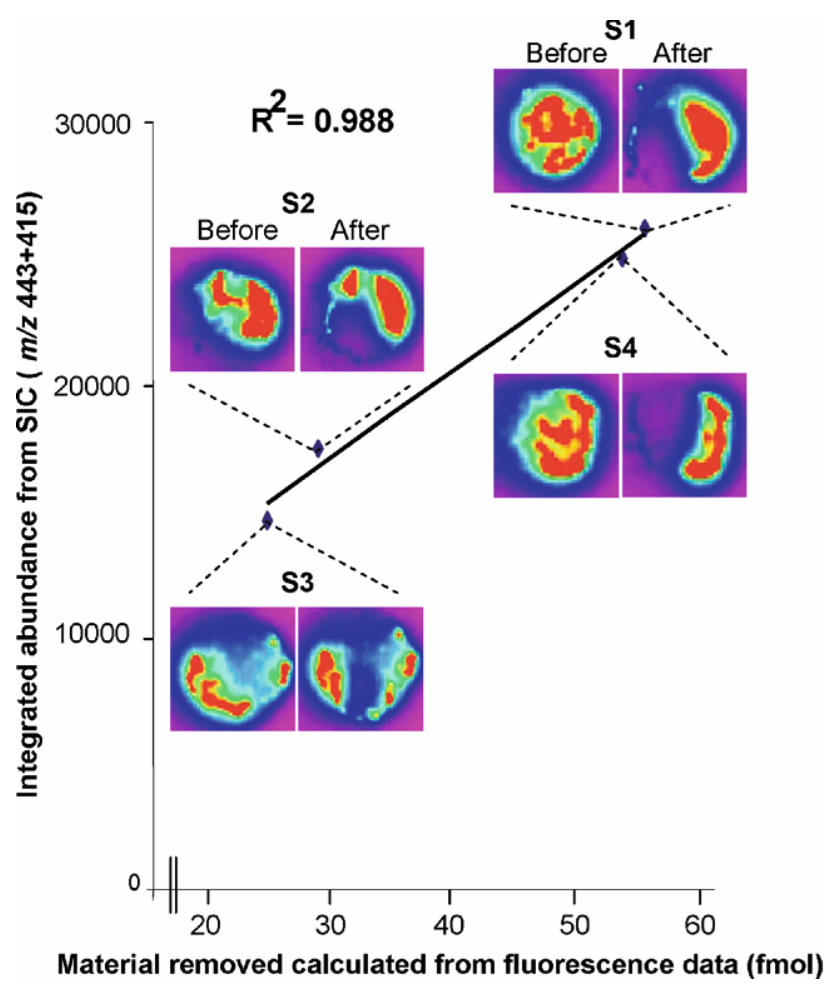

Figure 3. A plot of the integrated areas of the selected ion chromatograms $(m / z=443+415)$ versus the amount of material removed from the surface calculated from the fluorescence measurements. A strong correlation exists between these two parameters indicating the capability of fluorescence to probe the amount of material removed by DESI. Illustrated by the before and after DESI analysis fluorescent images, there were inhomogeneities across the surface of the spotted analyte. Thus, the MS signal abundance was dependent on where the spot of the DESI spray interacted with the analyte on the surface. These are magnified images and the analyte spot areas on the PTFE surface ranged from 2 to $8 \mathrm{~mm}^{2}$.

was presented as an example analyte and we emphasize that the same detectability (attomole detection) is not expected for all analytes, in part because of the varying response factors associated with DESI-MS analysis for different analytes. Currently, investigations are under way in our laboratory exploring these same fundamental aspects of DESI using fluorescent tags coupled to higher molecular weight analytes (such as peptides and proteins). It will be interesting to examine the relationship between the amount of material removed as a function of molecular weight as well as the effect of different surfaces. These planned studies will allow determination of the maximum molecular weight limit attainable by DESI-MS analysis, an important characteristic that has not yet been reported.

\section{Acknowledgments}

The authors thank R. Graham Cooks and his students for their aid in the design of the current DESI source used in these studies. In addition, they are especially grateful for the financial support received from the National Cancer Institute, National Institutes of Health (Grant R33 CA-105295), the W.M. Keck Foundation, and North Carolina State University.

\section{References}

1. Fenn, J. B.; Mann, M.; Meng, C. K.; Wong, S. F.; Whitehouse, C. M. Electrospray Ionization for Mass-Spectrometry of Large Biomolecules. Science 1989, 246, 64-71.

2. Fenn, J. B.; Mann, M.; Meng, C. K.; Wong, S. F.; Whitehouse, C. M. Electrospray Ionization-Principles and Practice. Mass Spectrom. Rev. 1990, 9, 37-70.

3. Karas, M.; Hillenkamp, F. Laser Desorption. Ionization of Proteins with Molecular Masses Exceeding 10,000 Daltons. Anal. Chem. 1988, 60, 2299-2301.

4. Tanaka, K.; Ido, Y.; Akita, S.; Yoshida, Y.; Yoshida, T. Protein and Polymer Analysis up to $m / z 100,000$ by Laser Ionization Time-of-Flight Mass Spectrometry. Rapid Commun. Mass Spectrom. 1988, 2, 151-153.

5. McEwen, C. N.; McKay, R. G.; Larsen, B. S. Analysis of Solids, Liquids, and Biological Tissues Using Solids Probe Introduction at Atmospheric Pressure on Commercial LC/MS Instruments. Anal. Chem. 2005, 77, 7826-7831.

6. Cody, R. B.; Laramee, J. A.; Durst, H. D. Versatile New Ion Source for the Analysis of Materials in Open Air under Ambient Conditions. Anal. Chem. 2005, 77, 2297-2302.

7. Shiea, J.; Huang, M. Z.; Hsu, H. J.; Lee, C. Y.; Yuan, C. H.; Beech, I.; Sunner, J. Electrospray-assisted Laser Desorption/Ionization Mass Spectrometry for Direct Ambient Analysis of Solids. Rapid Commun. Mass Spectrom. 2005, 19, 3701-3704.

8. Huang, M. Z.; Hsu, H. J.; Lee, L. Y.; Jeng, J. Y.; Shiea, L. T. Direct Protein Detection from Biological Media through Electrospray-assisted Laser Desorption Ionization/Mass Spectrometry. J. Proteome Res. 2006, 5, 1107-1116.

9. Sampson, J. S.; Hawkridge, A. M.; Muddiman, D. C. Generation and Detection of Multiply-charged Peptides and Proteins by Matrix-assisted Laser Desorption Electrospray Ionization (MALDESI) Fourier Transform Ion Cyclotron Resonance Mass Spectrometry. J. Am. Soc. Mass Spectrom. 2006, 17, 1712-1716.

10. Sampson, J. S.; Hawkridge, A. M.; Muddiman, D. C. Direct Characterization of Intact Polypeptides by Matrix Assisted Laser Desorption Electrospray Ionization (MALDESI) Quadrupole Fourier Transfrom Ion Cyclotron Resonance Mass Spectrometry. Rapid Commun. Mass Spectrom. 2007, 21, 1150-1154.

11. Takats, Z.; Wiseman, J. M.; Gologan, B.; Cooks, R. G. Mass Spectrometry Sampling under Ambient Conditions with Desorption Electrospray Ionization. Science 2004, 306, 471-473.

12. Cooks, R. G.; Ouyang, Z.; Takats, Z.; Wiseman, J. M. Ambient Mass Spectrometry. Science 2006, 311, 1566-1570.

13. Hu, Q. Z.; Talaty, N.; Noll, R. J.; Cooks, R. G. Desorption Electrospray Ionization Using an Orbitrap Mass Spectrometer: Exact Mass Measurements on Drugs and Peptides. Rapid Commun. Mass Spectrom. 2006, 20, 3403-3408.

14. Bereman, M. S.; Nyadong, L.; Fernandez, F. M.; Muddiman, D. C. Direct High-resolution Peptide and Protein Analysis by Desorption Electrospray Ionization Fourier Transform Ion Cyclotron Resonance Mass spray lonization Fourier Transform Ion Cyclotron Resonance Mas
Spectrometry. Rapid Commun. Mass Spectrom. 2006, 20, 3409-3411.

15. Van Berkel, G. J.; Ford, M. J.; Deibel, M. A. Thin-layer Chromatography and Mass Spectrometry Coupled Using Desorption Electrospray Ionization. Anal. Chem. 2005, 77, 1207-1215.

16. Weston, D. J.; Bateman, R.; Wilson, I. D.; Wood, T. R.; Creaser, C. S Direct Analysis of Pharmaceutical Drug Formulations Using Ion Mobility Spectrometry/Quadrupole-Time-of-Flight Mass Spectrometry Combined with Desorption Electrospray Ionization. Anal. Chem. 2005, $77,7572-7580$.

17. Williams, J. P.; Scrivens, J. H. Rapid Accurate Mass Desorption Electrospray Ionization Tandem Mass Spectrometry of Pharmaceutical Samples. Rapid Commun. Mass Spectrom. 2005, 19, 3643-3650.

18. Takats, Z.; Wiseman, J. M.; Cooks, R. G. Ambient Mass Spectrometry Using Desorption Electrospray Ionization (DESI): Instrumentation, Mechanisms and Applications in Forensics, Chemistry, and Biology. J. Mass Spectrom. 2005, 40, 1261-1275.

19. Van Berkel, G. J.; Kertesz, V. Automated Sampling and Imaging of Analytes Separated on Thin-layer Chromatography Plates Using Desorption Electrospray Ionization Mass Spectrometry. Anal. Chem. 2006, 78, 4938-4944.

20. Smith, R. D.; Loo, J. A.; Edmonds, C. G.; Barinaga, C. J.; Udseth, H. R. New Developments in Biochemical Mass-Spectrometry-Electrospray Ionization. Anal. Chem. 1990, 62, 882-899.

21. Zook, D. R.; Bruins, A. P. On Cluster Ions, Ion Transmission, and Linear Dynamic Range Limitations in Electrospray (Ionspray) Mass Spectrometry. Int. J. Mass Spectrom. Ion Processes 1997, 162, 129-147. 